\title{
Electrical and optical studies in polyaniline nanofibre--SnO nanocomposites
}

\author{
SMRITIMALA SARMAH and A KUMAR* \\ Department of Physics, Tezpur University, Tezpur 784 028, India
}

MS received 5 November 2008; revised 29 February 2012

\begin{abstract}
Polyaniline nanofibre-tin oxide $\left(\mathrm{PAni}_{-} \mathrm{SnO}_{2}\right)$ nanocomposites are synthesized and mixed with polyvinyl alcohol (PVA) as stabilizer to cast free-standing films. Composite films are characterized by X-ray diffraction studies (XRD), scanning electron microscopy (SEM), transmission electron microscopy (TEM), photoluminescence spectroscopy (PL) and UV-visible spectroscopy. XRD confirms the formation of PAni nanofibre-SnO $\mathrm{O}_{2}$ nanocomposite.

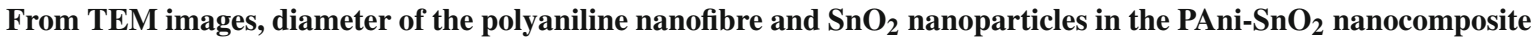
are found to be $20-60 \mathrm{~nm}$. SEM results show fibrous morphology of the PAni nanofibre and spherical morphology of polyaniline- $\mathrm{SnO}_{2}$ composites. The nanocomposites exhibit high relative photoluminescence intensity in violet as well as green-yellow region of visible spectrum. From electrical conductivity measurement, it is confirmed that PAni nanofibre-SnO $\mathrm{S}_{2}$ nanocomposite follows Mott's one-dimensional variable range hopping (VRH) model.
\end{abstract}

Keywords. Polyaniline nanofibres; tin oxide; nanocomposites; photoluminescence; TEM; UV-vis spectroscopy.

\section{Introduction}

Nanocomposites are a special class of materials originating from suitable combinations of two or more nanosized objects by some suitable techniques resulting in materials having unique physical properties and wide application potential in diverse areas (Gangopadhyay and De 2000). There is a growing interest in combining both organic and inorganic materials to synthesize nanocomposites for applications in electronics and photonics (Su and Kuramato 2000). Preparation of composites of polyaniline (PAni) has been considered to improve the processibility of PAni. These composites have the ability to enhance their material properties with desirable mechanical and physical characteristics (Raghavendra et al 2003). PAni is an unique and one of the most technologically promising conducting polymers because of its ease of preparation, low cost, high environmental stability, relatively stable electrical conductivity and especially for its very simple acid doping and base de-doping chemistry. Since the conductivity of PAni depends on both the oxidation state of the main polymer chain as well as the degree of protonation of the imine sites (MacDiarmid 2001), any interaction with PAni that alters either of these processes will affect its conductivity. PAni has a great variety of potential applications including anticorrosion coatings, batteries, sensors, separation membranes and antistatic coating (Chandrasekhar 1999). It is reported that PAni shows photoluminescence indicating existence of multiple electronic states including polaron bands, defect bands and con-

\footnotetext{
*Author for correspondence (ask@ tezu.ernet.in)
}

duction bands (Sharma et al 2006). PAni-semiconductor nanocomposites have become increasingly popular materials for applications in sensors, EMI shielding and antistatic coating due to enhanced processibility and protection of the semiconductor nanocomposites and conducting nature of host polymer. PAni is a good candidate for serving as the host matrix for inorganic semiconductors in optoelectronic applications because of its hole-conducting ability, when combined with the electron-conducting inorganic semiconductors, can increase the radiative electron-hole recombination rate so as to improve the optoelectronic performance of the inorganic semiconductors (Gaponik et al 2000). Semiconducting nanocomposites of PAni with inorganic semiconductors like cadmium sulphide (Lu et al 2005), cadmium telluride (Gaponik et al 1999), zinc oxide (Zheng et al 2002), zinc sulphide (Pant et al 2006) and titanium dioxide (Ganesan and Gedanken 2008) have been reported for diverse applications in optoelectronics.

In recent years, functional metal oxides have received increasing attention due to their unique physical properties (Ge et al 2006). Functional oxides have two structural characteristics: cations with mixed valence states and anions with deficiencies (vacancies). By varying either or both of these characteristics, the electrical, optical, magnetic and chemical properties can be tuned, giving the possibility of fabricating smart devices that utilize the semiconductivity, superconductivity, ferroelectricity and/or magnetism offered by the oxides (Wang and Kang 1998). Among the technologically promising functional metal oxides, tin oxide $\left(\mathrm{SnO}_{2}\right)$ has received a great deal of attention as a transparent conductor due to its applications in various opto-electronic devices including flat panel displays, photoconductors and solar cells 
(Amin et al 2001). $\mathrm{SnO}_{2}$ is a $n$-type semiconductor with a bandgap of $3.6 \mathrm{eV}$ at $300 \mathrm{~K}$, whereas PAni is a typical conductive polymer which is usually considered as a $p$-type material (Dey et al 2004).

In view of the foregoing, by synthesizing a nanocomposite of semiconducting material, $\mathrm{SnO}_{2}$ and conducting polymer, PAni, the electrical, optical and stability of the system can be enhanced. In the present work, we report the development of organic-inorganic PAni nanofibre- $\mathrm{SnO}_{2}$ hybrid nanocomposite in polyvinyl alcohol with a view to study its structural, electrical and optical properties.

\section{Experimental}

Aniline monomer (Sigma-Aldrich), ammonium peroxy disulphate (APS) (Merck), benzene (Sisco Research Lab), tin chloride dihydrate (Merck), ethanol (Changshu Yangyuan Chemical, China), tetrabutyl ammonium hydroxide (Merck), hydrochloric acid $(\mathrm{HCl})$ (Qualigens Fine Chemicals) and polyvinyl alcohol (PVA) $(\mathrm{BDH})$ (molecular weight = 11,000-31,000, degree of hydrolysis $=98-98.8 \%$ ) were used as received. $\mathrm{SnO}_{2}$ nanocolloids were prepared by a colloidal process described elsewhere (Dutta and De 2007). $0 \cdot 1 \mathrm{M} \mathrm{SnCl}_{2} \cdot 2 \mathrm{H}_{2} \mathrm{O}$ was dissolved in $50 \mathrm{ml}$ ethanol under constant magnetic stirring. $10 \mathrm{ml}$ double distilled (DD) water was added drop wise to the ethanol solution to keep chemical homogeneity. Addition of 0.01 M tetrabutyl ammonium hydroxide as a surfactant to the above solution produced smaller $\mathrm{SnO}_{2}$ colloidal particles. $0 \cdot 1 \mathrm{M} \mathrm{HCl}$ was added to keep $\mathrm{pH}$ of the solution below 4.

Polyaniline nanofibre- $\mathrm{SnO}_{2}$ nanocomposite was synthesized by interfacial polymerization technique. At first, $1 \mathrm{M}$ aniline was dissolved in $10 \mathrm{ml}$ benzene. $0.25 \mathrm{M}$ Ammonium peroxy disulphate (APS), $5 \mathrm{ml} \mathrm{SnO}_{2}$ colloids and $1 \mathrm{M} \mathrm{HCl}$ were dissolved in $10 \mathrm{ml}$ DD water. Both the solutions were added, in a glass vial to make an interface. As soon as the solutions were added, a green line appeared at the interface indicating the beginning of formation of PAni, which being hydrophilic moved into water allowing further polymerization at the interface and suppressing secondary growth (Huang 2006). PAni nanofibre- $\mathrm{SnO}_{2}$ composite was washed with DD water, filtered with Whatman filter paper and dried. $10 \mathrm{Wt} \%$ of nanocomposite powder was added to $20 \mathrm{ml} 4 \%$ PVA and stirred for $6 \mathrm{~h}$ at room temperature. Films were cast on clean glass plates and dried in a dessicator. PAni nanofibres without $\mathrm{SnO}_{2}$ colloids were also prepared by interfacial polymerization technique and $10 \mathrm{wt} \%$ of PAni nanofibres were mixed with $20 \mathrm{ml} 4 \%$ PVA to get PAni films.

XRD studies on PAni nanofibre, $\mathrm{SnO}_{2}$ and PAni nanofibre- $\mathrm{SnO}_{2}$ were carried out using Rigaku X-ray diffractometer (model MINIFLEX)-200. SEM micrograph was taken using JEOL-JSM 6390 LV scanning electron microscope. TEM images were taken using JEOL-TEM-100 CXII (carbon coated copper grids) in SAIF, Shillong. The UVvis spectroscopic measurements were conducted on aqueous dispersion of PAni nanofibre, $\mathrm{SnO}_{2}$ colloids and PAni nanofibre- $\mathrm{SnO}_{2}$ nanocomposite using UV-1700 spectrometer. The PL spectra were taken on films of $\mathrm{SnO}_{2}$, PAni nanofibre and PAni nanofibre- $\mathrm{SnO}_{2}$ nanocomposite using Perkin Elmer LS-55 fluorescence spectrometer. Electrical conductivity was measured using four-probe technique.

\section{Results and discussion}

\subsection{Structural and morphological studies}

Figure 1 shows X-ray diffraction patterns of (a) PAni nanofibre, (b) PAni nanofibre- $\mathrm{SnO}_{2}$ composite and (c) $\mathrm{SnO}_{2}$. In figure 1(a), the broad characteristic peak of polyaniline at $26^{\circ}$ indicates the formation of nanofibres. In figure 1(c) all the diffraction lines are assigned to the tetragonal rutile crystalline phase of tin oxide with peak positions at $2 \theta=11.55^{\circ}$ (110), $23.52^{\circ}(211), 35.48^{\circ}$ (222), $47.97^{\circ}$ (422). In figure 1(b), all the XRD peaks characteristics of $\mathrm{SnO}_{2}$ and PAni are present, which confirm the formation of PAni nanofibre$\mathrm{SnO}_{2}$ nanocomposite.

The diameters of PAni nanofibres and $\mathrm{SnO}_{2}$ nanoparticles were estimated using the Scherrer formula:

$$
D=0 \cdot 9 \lambda / \beta \cos \theta
$$

where $\lambda, \beta$ and $\theta$ are the X-ray wavelength $(1.5405 \AA$ for $\mathrm{CuK} \alpha$ ), the full width at half maximum (FWHM) of the diffraction peak, and Bragg diffraction angle, respectively. The polyaniline nanofibre diameters are in the range of 40$60 \mathrm{~nm}$ and the particle size of $\mathrm{SnO}_{2}$ particles is calculated to

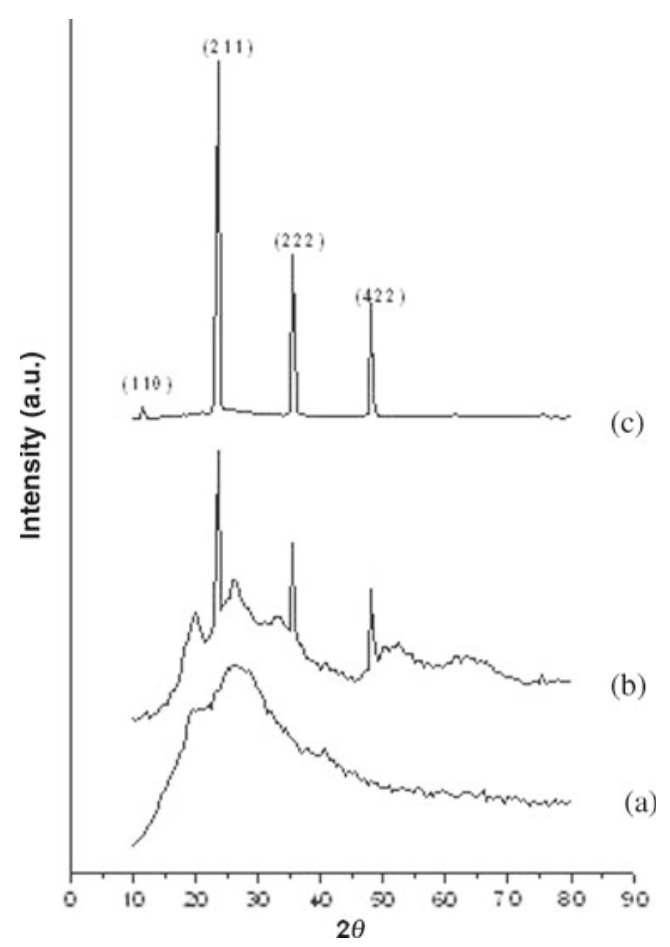

Figure 1. XRD spectra of (a) PAni nanofibre, (b) PAni nanofibre$\mathrm{SnO}_{2}$ nanocomposite and (c) $\mathrm{SnO}_{2}$. 
be in the range of $20-50 \mathrm{~nm}$, which is quite consistent with TEM results.

The scanning electron micrograph of PAni nanofibre$\mathrm{SnO}_{2}$ composite is shown in figure 2. The micrograph shows porous microstructure in which $\mathrm{SnO}_{2}$ nanoparticles are dispersed in fibrous PAni forming a nanocomposite. The transmission electron micrographs of PAni nanofibres and $\mathrm{PAni}_{-} \mathrm{SnO}_{2}$ nanocomposite are shown in figure 3(a and b), respectively. Figure 3(a) shows that the diameters of PAni nanofibres synthesized by interfacial polymerization technique are in the range of $40-60 \mathrm{~nm}$. In the PAni nanofibre$\mathrm{SnO}_{2}$ nanocomposite, polymerization of PAni takes place in the presence of spherical $\mathrm{SnO}_{2}$ nanoparticles of size 30$50 \mathrm{~nm}$ entrapping them inside PAni nanofibre chains, thus forming true nanocomposites as observed in figure 3(b).

\subsection{Optical properties}

Figure 4(a) shows UV-vis absorption spectrum of PAni nanofibres. The PAni nanofibres which are synthesized in the emeraldine salt form, in their 'compact coil' conformation, exhibits three prominent peaks (Xia et al 1995). The absorption peak at around $300 \mathrm{~nm}$ is attributed to the $\pi-\pi^{*}$ band transitions. The peak at around $440 \mathrm{~nm}$ is due to $\pi$-polaron band transitions and the peak at $800 \mathrm{~nm}$ is due to the polaron band $-\pi *$ transitions. The absorption bands obtained from the UV-visible spectra are consistent with that reported in the literature (Xia et al 1995). Although theoretical calculations predict that the bipolaron state is energetically more favoured than the polaron (Angelopolos et al 1988), it is widely agreed that polarons are the charge carriers responsible for high conductivity in PAni (Epstein et al 1987; Nakajima and Kawagoe 1989; Watanabe et al 1989). It has been proposed that the presence of coulombic interactions, dielectric screening and local disorder in the PAni lattice act to stabilize the delocalized polaron state (Bonnell and Angelopolos 1989).

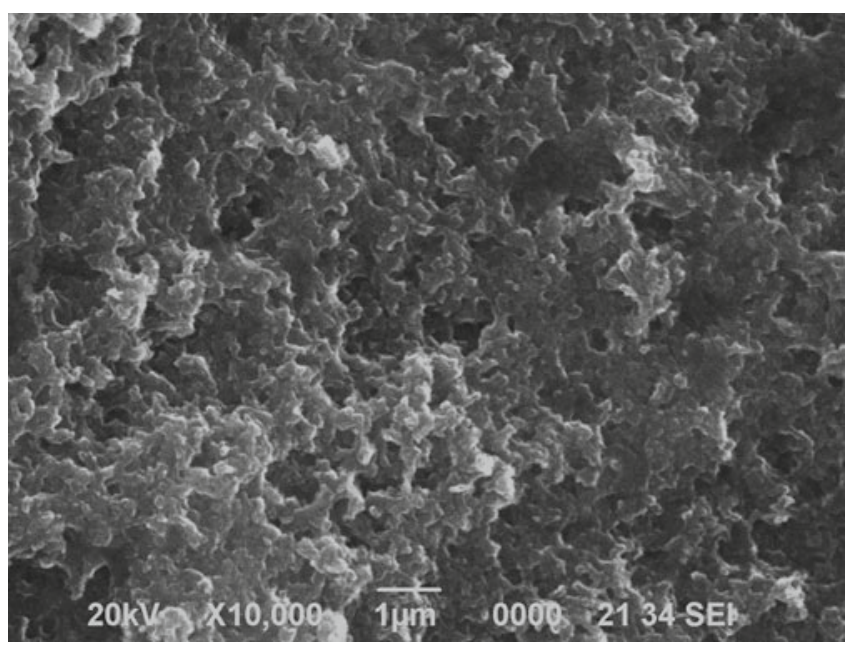

Figure 2. Scanning electron micrograph of PAni nanofibre- $\mathrm{SnO}_{2}$ nanocomposite.
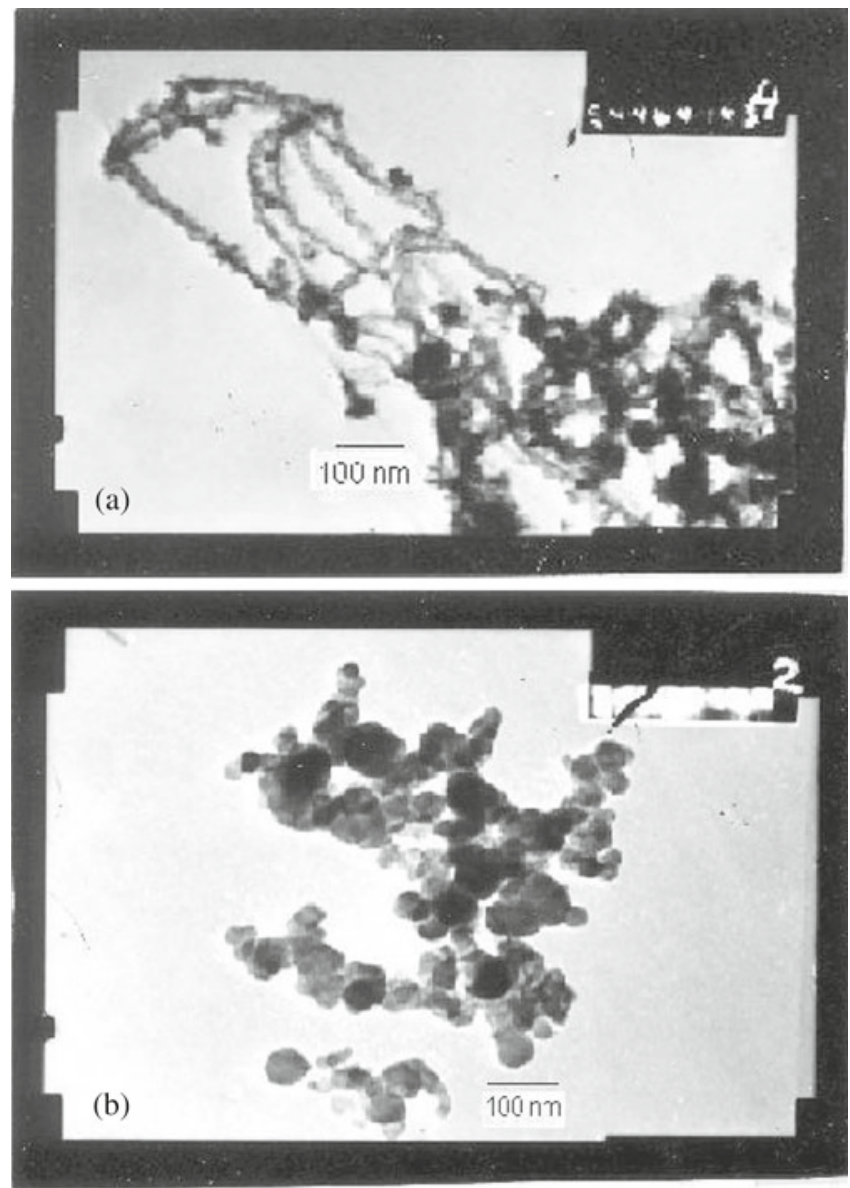

Figure 3. Transmission electron micrographs of (a) PAni nanofibre and (b) PAni nanofibre- $\mathrm{SnO}_{2}$ nanocomposite.

In the UV-vis spectra of pure $\mathrm{SnO}_{2}$ in figure 4(c), a strong peak is observed around $240 \mathrm{~nm}$ with a blue shift of energy of $0.83 \mathrm{eV}$ with respect to bulk $\mathrm{SnO}_{2}\left(E_{\mathrm{g}}=3.6 \mathrm{eV}\right)$ indicating particle size of $\mathrm{SnO}_{2}$ in the nanorange. In the $\mathrm{UV}$-vis spectra of PAni nanofibre- $\mathrm{SnO}_{2}$ nanocomposite in figure 4(b), all the peaks of PAni nanofibres as well as strong peak due to $\mathrm{SnO}_{2}$ are observed indicating the formation of PAni nanofibre- $\mathrm{SnO}_{2}$ nanocomposite.

Figure 5(a) shows PL spectrum of $\mathrm{SnO}_{2}$ excited with an excitation wavelength of $228 \mathrm{~nm}$. It shows a broad peak centred at $610 \mathrm{~nm}$. This peak might be related to the crystalline defects introduced during the growth of $\mathrm{SnO}_{2}$. The oxygen vacancies interact with interfacial tin vacancies leading to the formation of a considerable amount of trapped states within the bandgap, which results in a PL signal in $\mathrm{SnO}_{2}$ (He et al 2006). The PL spectra for PAni nanofibre and PAni nanofibre- $\mathrm{SnO}_{2}$ nanocomposite are shown in figures $5(\mathrm{~b}, \mathrm{c})$ with an excitation wavelength of $228 \mathrm{~nm}$. The peak at $414 \mathrm{~nm}$ in figure 5(b) for PAni arises due to transitions from the polaronic band to the $\pi$-band (HOMO) band structures of PAni (Sharma et al 2006). In figure 5(c) photoluminescence peaks at 503, 511 and $528 \mathrm{~nm}$ are observed. Figure 5(b, c) shows that the PL intensity is increasing in the PAni nanofibre$\mathrm{SnO}_{2}$ nanocomposite in both violet and green-yellow region 


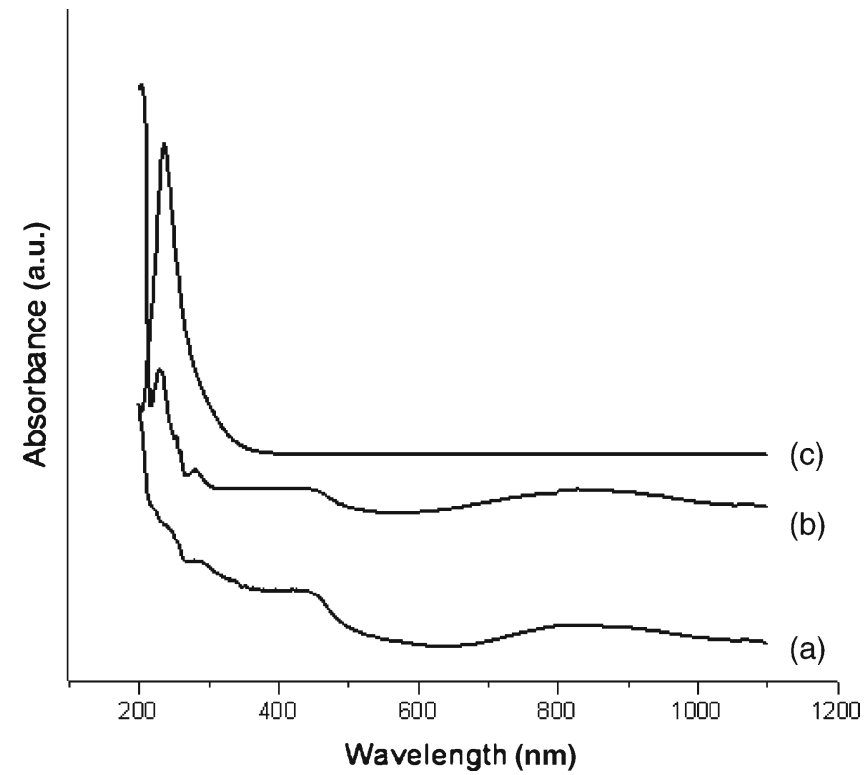

Figure 4. UV-vis spectra of (a) PAni nanofibre, (b) PAni nanofibre- $\mathrm{SnO}_{2}$ nanocomposite and (c) $\mathrm{SnO}_{2}$.

of visible spectrum, which may be attributed to formation of more density of states in the energy band upon the addition of $\mathrm{SnO}_{2}$. There are electron donating groups such as $=\mathrm{NH}$ in PAni and $\mathrm{SnO}_{2}$ is an electron conducting semiconductor. This combination enhances the electron mobility in the composites. This in turn favours the formation of singlet excitons. The singlet exciton states so formed decay radiatively to the ground state resulting in enhanced photoluminescence (Wise et al 1998). The relative intensity of $610 \mathrm{~nm} \mathrm{SnO}_{2}$ PL peak is considerably lower as compared to the relative peak intensity of PAni nanofibres, so it is not prominent in the PAni nanofibre-SnO $\mathrm{S}_{2}$ nanocomposite. Stafstorm and coworkers predicted the existence of lower energy transitions $(1.8$ and $2.8 \mathrm{eV})$ due to the polaron bands and higher energy transitions $(4.1 \mathrm{eV})$ from the upper defect band to the conduction band (Stafstorm et al 1987). Exact correlation with the results of Strafstorm is not possible in PAni nanofibre and PAni nanofibre- $\mathrm{SnO}_{2}$ nanocomposite due to disorder within the PAni nanofibrous structure and presence of dopant can also affect the energetic positions involved in practical situation. The presence of distinct peaks in the PL spectra indicates the possibility of the existence of multiple electronic states participating in the photo-excitation process (Sharma et al 2006).

\subsection{Electrical properties}

The temperature dependence of d.c. conductivity for PAni nanofibre and PAni nanofibre- $\mathrm{SnO}_{2}$ nanocomposite is shown in figure 6. The figure shows that both PAni nanofibres and PAni nanofibre- $\mathrm{SnO}_{2}$ nanocomposite samples are semiconducting in nature and the conductivity is higher for PAni nanofibre- $\mathrm{SnO}_{2}$ nanocomposite as compared to that for
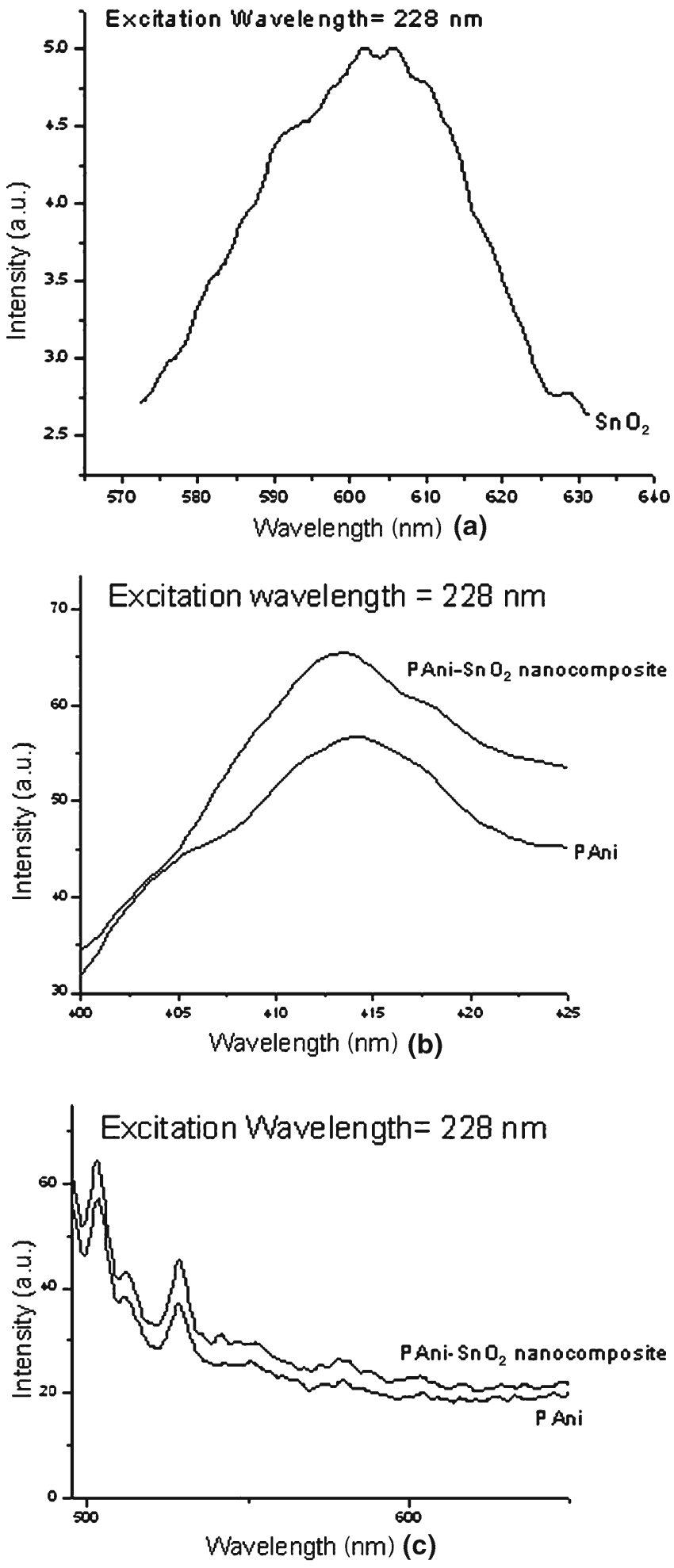

Figure 5. PL spectra of (a) $\mathrm{SnO}_{2}$, PL spectra of PAni nanofibre and PAni nanofibre- $\mathrm{SnO}_{2}$ nanocomposite in (b) violet region and (c) green-yellow region.

PAni nanofibre. The room temperature d.c. conductivity for PAni nanofibre and PAni nanofibre- $\mathrm{SnO}_{2}$ composite are calculated to be $7.19 \times 10^{-4} \mathrm{Scm}^{-1}$ and $1.22 \times 10^{-3} \mathrm{Scm}^{-1}$, 


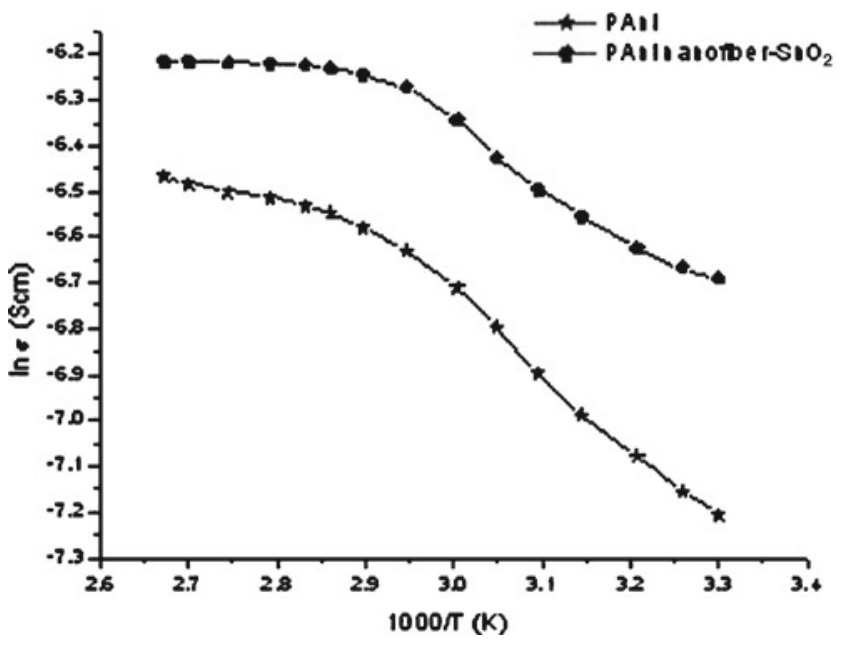

Figure 6. Temperature dependence of $\sigma$ electrical conductivity (ln $\sigma$ vs $10^{3} / T$ ) of PAni nanofibre and PAni nanofibre- $\mathrm{SnO}_{2}$ nanocomposite.

respectively. Due to introduction of $\mathrm{SnO}_{2}$, electronic density of states are increasing in the polaronic band between the $\pi$ $\pi *$ HOMO-LUMO structure of PAni at the PAni nanofibre$\mathrm{SnO}_{2}$ interface. This results in an increase in conductivity not only by increasing the carrier concentration but also by increasing the mobility which renders the interchain charge transport more efficient (Luthra et al 2003).

The temperature dependence of conductivity can be best fitted within the experimental error with one-dimensional phonon assisted Mott's variable range hopping (VRH) model:

$$
\sigma=\sigma_{0} \exp \left[-\left(T / T_{0}\right)^{1 / 2}\right]
$$

where pre-exponential factor $\sigma_{0}=e^{2} N\left(E_{\mathrm{F}}\right) R v_{\mathrm{ph}}, R$ the average hopping distance:

$$
R=\left[8 / 9 \pi \alpha N\left(E_{\mathrm{F}}\right) k T\right]^{-1 / 2} \text { and } T_{0}=8^{3} \alpha^{3} / 9 \pi k N\left(E_{\mathrm{F}}\right),
$$

where $N\left(E_{\mathrm{F}}\right)$ is the electronic density of states at the Fermi energy, $\alpha$ the inverse localization length (spatial extension of localized wave function), $e$ the electronic charge, $v_{\mathrm{ph}}$ the typical frequency $\left(\approx 10^{13} \mathrm{~Hz}\right), T_{0}$ the characteristic Mott temperature which corresponds to the hopping barrier for charge carriers (also known as the pseudo activation energy) and measures the degree of disorder (Mott and Davis 1979; Roth and Carroll 2004). From the linear curve fitting of $\ln \sigma$ vs $T^{-1 / 2}, \sigma_{0}$ and $T_{0}$ are found to be $4.197 \times 10^{-3} \mathrm{Scm}^{-1}$ and $78.14 \mathrm{~K}$, respectively for PAni nanofibre and $4.279 \times 10^{-3}$ $\mathrm{Scm}^{-1}$ and $41.25 \mathrm{~K}$ for PAni nanofibre- $\mathrm{SnO}_{2}$ nanocomposite. The size of hopping barrier, $T_{0}$, is smaller in the PAni nanofibre- $\mathrm{SnO}_{2}$ nanocomposite as compared to PAni nanofibre. This enhances the hopping rate between adjacent localized states and leads to an increase in electrical conductivity in PAni nanofibre- $\mathrm{SnO}_{2}$ nanocomposite (Mott and Davis 1979).

\section{Conclusions}

Polyaniline nanofibre- $\mathrm{SnO}_{2}$ nanocomposites are synthesized by interfacial polymerization technique. XRD and TEM confirm the formation of nanocomposites with diameters of PAni nanofibre and $\mathrm{SnO}_{2}$ nanoparticles in the range of 20$60 \mathrm{~nm}$. PL spectra shows an increase in relative photoluminescence intensity in PAni nanofibre- $\mathrm{SnO}_{2}$ nanocomposite in violet and green-yellow region of visible spectrum as compared to that for pure PAni nanofibre. Temperature dependence of electrical conductivity of the PAni nanofibre$\mathrm{SnO}_{2}$ nanocomposite fits the one-dimensional Mott's variable range hopping model. From this model, it can be concluded that the increase in electrical conductivity in the PAni nanofibre- $\mathrm{SnO}_{2}$ nanocomposite is due to decrease of hopping barrier as a result of more number of density of states introduced by $\mathrm{SnO}_{2}$ in the PAni nanofibre- $\mathrm{SnO}_{2}$ interface. This nanocomposite can be used to fabricate optoelectronic devices with enhanced electrical and optical properties.

\section{Acknowledgements}

Financial support of UGC through project No F.31$7 / 2005$ (SR) is greatly acknowledged. Authors are thankful to SAIF, NEHU, Shillong, for transmission electron microscopic analysis.

\section{References}

Angelopolos M, Asturias G E, Ermer S P, Scherr E M, MacDiarmid A G, Akhtar M, Kiss Z and Epstein A J 1988 Mol. Cryst. Liq. Cryst. 160151

Amin N, Isaka T, Yamada A and Konagai M 2001 Sol. Energy Mater. Sol. Cells 67195

Bonnell D A and Angelopolos M 1989 Synth. Met. 33301

Chandrasekhar P 1999 Conducting polymers, fundamentals and applications: A practical approach (Boston: Kluwer Academic) p. 760

Dey A, De S, De A and De S K 2004 Nanotechnology 151277

Dutta K and De S K 2007 J. Phys. D: Appl. Phys. 40734

Epstein A J et al 1987 Synth. Met. 18303

Ganesan R and Gedanken A 2008 Nanotechnology 19435709

Gangopadhyay R and De A 2000 Chem. Mater. 12608

Gaponik N P, Talapin D V, Rogach A L and Eychmuller A 2000 J. Mater. Chem. 102163

Gaponik N P, Talapin D V and Rogach A L 1999 Phys. Chem. Chem. Phys. 11787

Ge J P, Wang J, Zhang H, Wang X, Peng X and Li Q Y D 2006 Sens. Actuators Chem. B113 937

He H Jr, Wu T H, Hsin C L, Li K M, Chen L J, Chueh Y L, Chou L J and Wang Z L 2006 Small 2116

Huang J 2006 Pure Appl. Chem. 7815

Lu X, Gao H, Chen J, Chao D, Zhang W and We Y 2005 Nanotechnology 16113

Luthra V, Singh R, Gupta S K and Mansingh A 2003 Curr. Appl. Phys. 3219

MacDiarmid A G 2001 Synth. Metals 12511

Mott N F and Davis E A 1979 Electronic processes in noncrystalline materials (Oxford: Clarendon) p. 157 
Nakajima T and Kawagoe T 1989 Synth. Met. 28 C629

Pant H C, Patra M K, Negi S C, Bhatia A, Vadera S R and Kumar N 2006 Bull. Mater. Sci. 29379

Raghavendra S C, Khasim S, Revanasiddappa M, Prasad M V N A and Kulkarni A B 2003 Bull. Mater. Sci. 26733

Roth S and Carroll D 2004 One-dimensional metals (Weinheim: Wiley-VCH: Verlag GmbH \& Co. KGaA) 2nd ed., p. 141

Sharma M, Kaushik D, Singh R R and Pandey R K 2006 J. Mater. Sci.: Mater. Electron. 17537

Stafstorm S, Breadas J L, Epstein S J, Woo H S, Tanner D B, Huang W S and MacDiarmid A G 1987 Phys. Rev. Lett. 59 1464
Su S J and Kuramato N 2000 Synth. Metals 114147

Wang Z L and Kang Z C 1998 Functional and smart materials (New York: Plenum) p. 111

Watanabe A, Mori K, Mikuni M, Nakamura Y and Matsuda M 1989 Macromolecules 223323

Wise D L, Wnek G E, Trantlolo D, Cooper T M and Gresser J D 1998 Photonic polymer systems-Fundamentals, methods and applications (New York: Marcel Dekker, Inc.)

Xia Y, Wiesinger J M, MacDiarmid A G and Epstein A J 1995 Chem. Mater. 7443

Zheng Z X, Xi Y Y, Dong P, Huang H G, Zhou J Z, Wu L L and Lin Z H 2002 Phys. Chem. Commun. 56 